\title{
Isolated pulmonary metastasis from prostate Adenocarcinoma: Diagnosis by Fine Needle Aspiration Cytology
}

\author{
Anju Khairwa', Gupta Nalini', Singhal Manphool'2, Kumar Santosh ${ }^{3}$, Rajwanshi Arvind ${ }^{1}$ \\ ${ }^{1}$ Departments of Cytology and Gynecological Pathology, ${ }^{2}$ Department of Radiodiagnosis, ${ }^{3}$ Department of Urology, Postgraduate Institute of \\ Medical Education and Research, Chandigarh
}

\section{A B S T R A C T}

Prostate cancer usually metastasizes to regional lymph nodes and bone followed by distant organs. Here, we present a case of elderly man who had isolated respiratory symptoms and finally turned out to be metastasis from prostate cancer. Rarity of isolated pulmonary metastases of prostate cancer without bone and lymph node involment prompted us to report this case which was diagnosed by CT guided FNAC.

Access this article online

Website:

http://nepjol.info/index.php/AJMS

DOI: 10.3126/ajms.v6i2.11103

Key words: Prostate carcinoma, Pulmonary metastasis, Fine needle aspiration cytology, Immunohistochemistry

\section{INTRODUCTION}

Prostate cancer commonly metastasizes to bone and regional lymph nodes and uncommonly to lung, liver, and brain. Prostate adenocarcinoma leads to osteoblastic type of osseous metastasis and lymph node metastases followed by pulmonary metastases. The role of fine needle aspiration cytology (FNAC) for diagnosis of metastatic malignancies is well established in the literature. ${ }^{1}$ Isolated pulmonary metastases of prostate cancer without bone and lymph node involment is rarely described in literature. Fabozzi et al reported radiologic evidence of lung metastases in 48 out of 1290 patients, with 11 (0.86\%) being a solitary pulmonary nodule. ${ }^{2}$ Here, we discuss such a case which was diagnosed by CT guided FNAC.

\section{CASE HISTORY}

A 62-year-old man presented with cough and progressive dysponea without any associated fever, weight loss, or night sweats for the last three months. He was a known case of carcinoma prostate, diagnosed six years back by prostatic biopsy that showed an adenocarcinoma occupying $30 \%$ of biopsy with Gleason score $3+4=7$. Contrast enhanced CT (CECT) scan at that time showed bulky prostate with seminal vesicle involvement. Bone metastasis was ruled out by normal bone scan then. The patient was treated with radiotherapy and received GnRH agonist for two years. Prostate Specific Antigen (PSA) was $0.8 \mathrm{ng} / \mathrm{ml}$ after one year of this treatment. Positron Emission Tomography(PET) scan was done at that time and showed no evidence of abnormal hyper metabolic lesion anywhere in the body. Patient developed bilateral gynaecomastia post-chemotherapy. During current illness, there was no evidence of anemia, leukocytosis, hypoalbuminemia, electrolyte imbalance or abnormal liver function tests. His chest $\mathrm{x}$-ray showed "extensive cannon-ball type lesions" involving both lungs. The patient underwent an enhanced computed tomography (CT) scan of the chest, abdomen, and pelvis. The CT scan of chest showed a diffuse nodular type multiple lesions ranging in size from few millimeters to $3 \mathrm{~cm}$ in all segments of lung. CT scan of the abdomen and pelvis did not reveal any retroperitoneal, para-aortic, and pelvic lymph node enlargement and there were no lesions in

Address for Correspondence:

Dr Nalini Gupta, Associate Professor, Department of Cytology and Gynecologic Pathology, Postgraduate Institute of Medical Education and

Research, Chandigarh, India

E-mail: nalini203@gmail.com; Phone: +91-1722755114; Fax: +91-172-2744401

(C) Copyright AJMS 
liver, spleen, pancreas, and adrenals. MRI of pelvis and lumbo-sacral spine showed small prostate gland with abnormal hypointensity in left seminal vesicle and left postero-lateral corner of prostate suggestive of tumor infiltration in prostate and left seminal vesicle. Spine did not reveal any abnormal signal or lesion suggestive of metastasis. PET scan of whole body revealed Fluorodeoxyglucose (FDG) avid multiple nodules in bilateral lungs. 99m'Tc-methylene diphosphonate (MDP) bone scan and serum PSA levels were normal. The presence of the above findings raised the possibility of

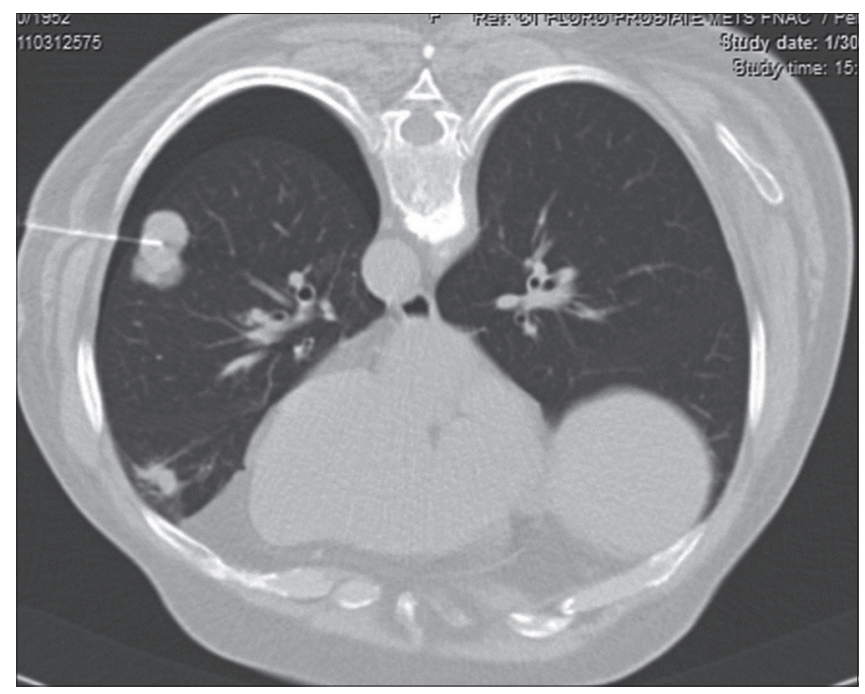

Figure 1: Axial CT guided FNAC image in prone position with needle in-situ in one of the metastatic lesion in right lower lobe. Note minimal pneumothorax.

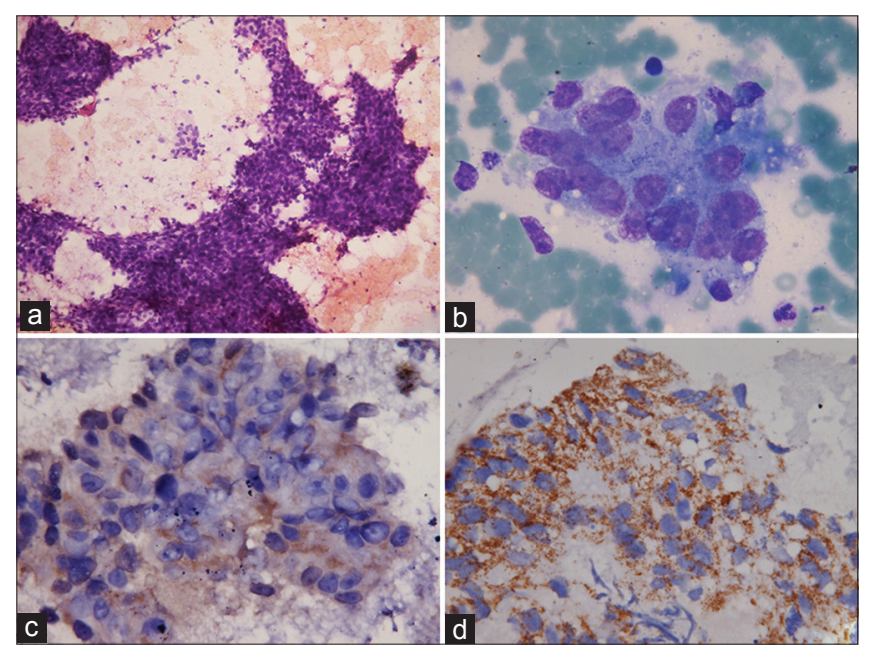

Figure 2: A panel of microphotographs; 2a- cellular smears showing clusters and singly scattered tumor cells (H\&E, 20X); 2b- Tumor cells showing nuclear pleomorphism, coarse chromatin, prominent nucleoli and moderate amount of cytoplasm (MGG, 100X); 2c- Tumor cells showing focal cytoplasmic positivity for PSA (IHC for PSA, 40X); 2d- Tumor cells showing strong granular cytoplasmic positivity for AMACR (IHC for AMACR, 40X) either a primary lung cancer or diffuse lung metastases from prostate adenocarcinoma.

Patient underwent CT-guided FNAC from the largest lung nodule (Figure 1). FNAC smears were cellular and showed many clusters and singly scattered tumor cells (Figure 2a). The tumor cells were having moderately pleomorphic nuclei, coarse chromatin, prominent nucleoli and moderate amount of vacuolated cytoplasm (Figure 2b). The cytomorphology was of adenocarcinoma. To confirm whether adenocarcinoma was primary lung or metastatic adenocarcinoma, immunohistochemistry (IHC) was done on cell block. IHC panel was applied for lung and prostate. The tumor cells showed focal positivity for PSA (Figure 2c) and EMA, strong granular cytoplasmic positivity for AMACR (Figure 2d), CK7 focally positive and negative for TTF-1 (Figure 3) and p63. Overall features were of metastatic prostate adenocarcinoma in lung.

\section{DISCUSSION}

We report a metastatic adenocarcinoma in lung from prostate using FNAC and confirmed by IHC. FNAC is a useful tool for diagnosing the metastatic malignancies involving common as well as unusual sites. ${ }^{1}$ Pulmonary metastases in prostate cancer usually occur following osseous and/or lymph node metastasis. The index case had isolated pulmonary metastases after treatment for primary prostatic cancer. Prostate cancer constitutes about one-fourth of newly diagnosed carcinomas in males. ${ }^{3}$ The most common metastatic sites are bone and/or lymph nodes followed by pulmonary metastatsis. ${ }^{4,5}$ Bone metastasis in prostate carcinoma is usually osteoblastic type and commonly involves spine

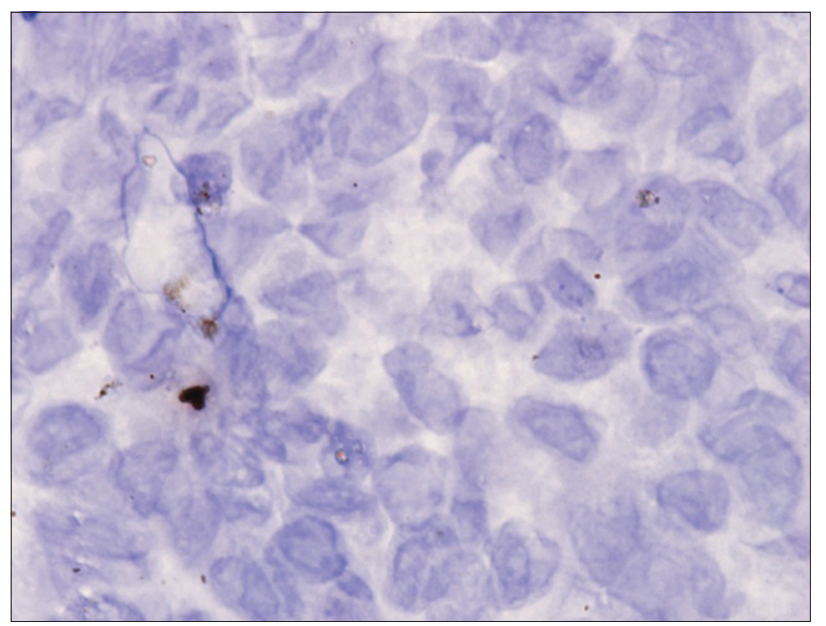

Figure 3: Tumor cells negative for TTF-1 (IHC for TTF-1, 100X) 
and pelvis. ${ }^{6}$ About $5-27 \%$ patients may have clinically apparent pulmonary metastases at initial presentation of prostate cancer and the rate of pulmonary metastasis increases to $23-74 \%$ at autopsy. ${ }^{2-4}$ Pulmonary metastases without lymphadenopathy and without any concomitant osseous or multi-organ involvement, like the present case, is rarely described in literature. Tohfe et $a \Gamma$ reported a case of prostate cancer who presented initially with symptomatic metastatic lung lesions, but unlike the present case, he also had lymph nodal involvement and prostate carcinoma associated with enlarged prostate and high serum PSA levels. Index case had normal PSA levels and no prostatic enlargement and did not have any bone or lymph node involvement. There are a few case reports of patients with isolated peritoneal or pulmonary metastasis from prostate cancer without bone metastasis accompanied by ascites. ${ }^{8,9}$ To best of our knowledge, such presentation of isolated lung metastasis from prostate has not been described in literature previously. Radiological appearance of pulmonary metastases in prostate cancer are commonly either lymphangitic or nodular, with the former being more frequent since it is apparently a result of direct invasion of lung lymphatics, while the nodular pattern being due to hematogenous spread. ${ }^{3,6}$ The index patient had a nodular pattern on CT which is suggestive of hematogenous spread and it is usually associated with other forms of hematogenous spread including bone involvement. ${ }^{10,11}$ There is a possibility of small recurrence in prostate that micro-metastasized in lung via hematogenous spread. This pattern of spread opens the door for more research about the pathophysiology of the mechanisms underlying distant metastases in prostate adenocarcinoma. Such micro-metastasis is difficult to detect by imaging techniques and IHC may be helpful in these cases. ${ }^{12}$ We confirmed lung metastasis from prostate origin by IHC on FNAC in the index case. Although IHC for PSA was weakly positive, IHC for AMACR showed strong cytoplasmic granular positivity, indicating the same to be a better marker for prostate cancer. Tumor cells were negative for TTF-1, which helps to exclude a primary lung adenocarcinoma. FNAC with IHC on cell block is an important tool to diagnose metastatic malignancies involving unusual sites. Hormonal drugs given in the setting of castration resistant prostate cancer are estrogen, antiandrogen and ketoconazole. ${ }^{13}$ Docetaxel is an important agent in hormone resistant prostate cancer (HRPC). Newer drugs approved for HRPC like abiraterone and enzalutamide were initially approved only after this chemotherapy had failed. Cabazitaxel, a microtubule inhibitor related to docetaxel, has also been approved in the post-docetaxel setting. As per American urological association guidelines, the present symptomatic pulmonary metastasis patient was started on docetaxel chemotherapy. Visceral and symptomatic metastasis is need to be treated aggressively in patient with good performance status. Symptom at HRPC with poor performance status should be offer other safer hormonal alternative or symptomatic treatment. On follow-up, this patient responded well after chemotherapy.

To conclude, this report emphasizes the importance of suspecting prostate cancer in men presenting with secondary lesions in the lung even in the absence of bone and lymph node metastases. FNAC features of metastatic malignancies and IHC have crucial role for confirming such lesions.

\section{KEY MESSAGES}

This report emphasizes the importance of suspecting prostate cancer in men presenting with secondary lesions in the lung even in the absence of bone and lymph node metastases. FNAC features of metastatic malignancies and IHC have crucial role for confirming such lesions.

\section{REFERENCES}

1. Elsheikh TM, Herzberg AJ and Jan F. Silverman JF. Fine needle aspiration cytology of metastatic malignancies involving unusual sites. Fine needle aspiration cytology of metastatic malignancies: Vol.108.No.4 (Suppl.1).

2. Fabozzi SJ, Schellhammer PF and el-Mahdi AM. Pulmonary metastases from prostate cancer. Cancer 1995; 75:2706-2709.

3. Jemal A, Siegel R, Ward E, Hao Y, Xu J, Murray T, et al. Cancer Statistics, 2008. CA Cancer J Clin 2008; 58:71-96.

4. Bubendorf L, Schöpfer A, Wagner U, Sauter G, Moch H, Willi N, et al. Metastatic patterns of prostate cancer: An autopsy study of 1,589 patients. Hum Pathol 2000; 31:578-583.

5. Saitoh H, Yoshida K, Uchijima Y, Kobayashi N, Suwata J and Kamata S. Two different lymph node metastatic patterns of a prostate cancer. Cancer 1990; 65:1843-1846.

6. Kakhki VR, Anvari K, Sadeghi R, Mahmoudian AS and Torabian-Kakhki M. Pattern and distribution of bone metastases in common malignant tumors. Nucl Med Rev Cent East Eur 2013; 16:66-69.

7. Tohfe M, Baki SA, Saliba W, Ghandour F, Ashou R, Ghazal G, et al. Metastatic prostate adenocarcinoma presenting with pulmonary symptoms: A case report and review of the literature. Cases Journal 2008; 1:316.

8. Zagouri F, Papaefthimiou M, Chalazonitis AN, Antoniou N, Dimopoulos MA and Bamias A. Prostate cancer with metastasis to the omentum and massive ascites: A rare manifestation of a common disease. Onkologie 2009; 32:758-761.

9. Wallis CJD, English JC and Goldenberg SL. The role of resection of pulmonary metastases from prostate cancer: A case report and literature review. Can Urol Assoc J 2011; 5:E104-E108. 
10. Apple JS, Paulson DF, Baber C and Putman CE. Advanced prostatic carcinoma: Pulmonary manifestations. Radiology 1985; 154:601-604.

11. Hofland CA and Bagg MD. An isolated pulmonary metastasis in prostate cancer. Mil Med 2000; 165:973-974.

12. Debus E, Moll R, Franke WW, Weber $\mathrm{K}$ and Osborn M.
Immunohistochemical distinction of human carcinoma by cytokeratin typing with monoclonal antibodies. Am J Pathol 1984; 114:121-130.

13. Gomella LG, Petrylak DP and Shavegan B. Current management of advanced and castration resistant prostate cancer. Can J Urol 2014; 21 (2 Supp 1):1-6.

\footnotetext{
Authors Contribution:

AK - Conceived and designed the study, made the first draft of the manuscript; AK, NG, AR - Concept and design of the study, analysis and interpretation of data; AK, NG, MS, SK, AR - Literature searches and manuscript preparation; All authors reviewed and approved of the final manuscript.

Source of Support: Nil, Conflict of Interest: None declared.
} 